\title{
Response to the comment on « EBIC contrast theory of dislocations : intrinsic recombination properties »
}

\author{
J. L. Farvacque and B. Sieber \\ Laboratoire de Structure et Propriétés de l'Etat Solide, URA 234, Bâtiment C6, Université des Sciences et \\ Techniques de Lille Flandre-Artois, 59655 Villeneuve d'Ascq Cedex, France
}

(Received 5 July 1990, accepted 12 July 1990)

\begin{abstract}
Résumé. - Nous démontrons, point par point, que les objections faites par Donolato et Pasemann sur la validité des calculs publiés dans l'article «Théorie EBIC du contraste des dislocations: propriétés de recombinaison intrinsèque » sont erronées et probablement dues à une incompréhension de notre approche physique du problème. En particulier, dans notre analyse, le contraste de la dislocation s'annule bien lorsque son activité électrique disparaît, contrairement à ce qu'ils affirment dans le résumé de leur commentaire.

Abstract. - We demonstrate, step by step, that the objections made by Donolato and Pasemann on the validity of calculations we have published in " EBIC contrast theory of dislocations : intrinsic recombination properties " are issued from a misunderstanding of our physical approach of the problem. On the opposite to what they claim in the abstract of their comment, the dislocation contrast is indeed equal to zero when the dislocation recombination activity vanishes.
\end{abstract}

The comment by Donolato and Pasemann [1] on the calculation of the EBIC contrast associated with the electrical field of a dislocation [2] shows that our approach can be misunderstood. Thus, in the following, we give a more detailed explanation of our physical model which allows to deduce expression (18) from expression (11) of the original paper [2].

In absence of any defect the excess carrier distribution $p_{0}(\mathbf{r})$ is solution of the following diffusion equation :

$$
D \Delta p_{0}(\mathbf{r})=\frac{p_{0}(\mathbf{r})}{\tau}-g(\mathbf{r})
$$

It has been explained in our paper that the disloca ion recom ina $\mathrm{i}$

scribed by the existence of its electrical field which is permanent despite the capture of minority carriers and even if its extension is selfconsistently reduced by the saturation effect as proposed by Wilshaw [3]. Thus, in presence of such a defect, the diffusion equation to be solved becomes :

$$
D \Delta p(\mathbf{r})=\frac{p(\mathbf{r})}{\tau}-g(\mathbf{r})+\operatorname{div}[\sigma \mathbf{E}]
$$

where $\mathbf{E}$ is the dislocation electrical field and $p(\mathbf{r})$ the new carrier distribution.
Using the Green function associated with equations (1) and (2) and the boundary conditions of the problem, it is easy to show, following an original calculation proposed by Donolato, that the variation $\delta I$ of the collected current, induced by the dislocation, corresponds to :

$$
\delta I=-\int_{V_{\text {crystal }}} \mathrm{e}^{-z / L} \operatorname{div}[\sigma \mathbf{E}] \mathrm{d} \tau
$$

where $L$ is the diffusion length. This last relation corresponds to formula (11) of our original paper.

As we mentionned in paper [2], the dislocation electric field is a space continuous but rapidly decreasing function of the distance $r$ from the dislocation line. It is for instance escri e y a $K_{1}\left(\lambda_{\mathrm{g}} r\right)$ function in the Debye-Hückel screnning approximation, where $K_{1}$ is a modified Bessel function of the first kind and $\lambda_{\mathrm{g}}$ the generalized DebyeHückel wavelength (see for instance [4]).

The dislocation can thus be considered as surrounded by a volume $V_{\mathrm{d}}$ outside of which the dislocation electric field is rapidly negligible (but not yet neglected). Considering such a volume $V_{\mathrm{d}}$, equation (1) can then be written :

$$
\delta I=-\int_{V_{\mathrm{d}}} \mathrm{e}^{-z / L} \operatorname{div}[\boldsymbol{\sigma E}] \mathrm{d} \tau+\delta I_{1}
$$


where :

$$
\delta I_{1}=-\int_{V_{\mathrm{ext}}} \mathrm{e}^{-z / L} \operatorname{div}[\sigma \mathbf{E}] \mathrm{d} \tau
$$

and where $V_{\text {ext }}$ represents the crystal volume outside $V_{\mathrm{d}}$. Writting the excess carrier distribution $p(\mathbf{r})$ in presence of the dislocation electric field under the form $p(\mathbf{r})=p_{0}(\mathbf{r})+\delta p(\mathbf{r})$, one can therefore replace $\operatorname{div}[\sigma \mathbf{E}]$ in expression (4) by its equivalent expression deduced from (1) and (2) :

$$
\operatorname{div}[\sigma \mathbf{E}]=\mathrm{e}\left\{D \Delta \delta p(\mathbf{r})-\frac{p(\mathbf{r})-p_{0}(\mathbf{r})}{\tau}\right\}
$$

It gives :

$$
\begin{aligned}
\delta I=- & \frac{e}{\tau} \int_{V_{\mathrm{d}}} \mathrm{e}^{-z / L}\left\{p_{0}(\mathbf{r})-p(\mathbf{r})\right\} \mathrm{d} \tau- \\
& -e D \int_{V_{\mathrm{d}}} \mathrm{e}^{-z / L} \operatorname{div}[\operatorname{grad} \delta p(\mathbf{r})] \mathrm{d} \tau+\delta I_{1} .
\end{aligned}
$$

Using the identity :

$$
\begin{aligned}
\mathrm{e}^{-z / L} \operatorname{div}[\operatorname{grad} \delta p(\mathbf{r})]= & \\
& \operatorname{div}\left[\mathrm{e}^{-z / L} \operatorname{grad} \delta p(\mathbf{r})\right] \\
& -\operatorname{grad} \delta p(\mathbf{r}) \cdot \operatorname{grad}\left(\mathrm{e}^{-z / L}\right)
\end{aligned}
$$

and since any of the functions introduced in the present calculation is continuous and derivable at any point, one of the volume integral can be transformed into a flux integral with help of Ostrogradsky's theorem : we easily obtain :

$$
\begin{aligned}
\delta I & =-\frac{e}{\tau} \int_{V_{\mathrm{d}}} \mathrm{e}^{-z / L} p_{0}(\mathbf{r}) \mathrm{d} \tau- \\
& -e D \int_{S_{\mathrm{d}}} \mathrm{e}^{-z / L} \operatorname{grad} \delta p(\mathbf{r}) \mathrm{d} \tau+\delta I_{1}+\delta I_{2}+\delta I_{3}
\end{aligned}
$$

with :

$$
\begin{aligned}
& \delta I_{1}=-\int_{V_{\mathrm{ext}}} \mathrm{e}^{-z / L} \operatorname{div}[\sigma \mathbf{E}] \mathrm{d} \tau \\
& \delta I_{2}=-\int_{V_{\mathrm{d}}} \delta \mathbf{j}_{\mathrm{d}} \operatorname{grad}\left(\mathrm{e}^{-z / L}\right) \\
& \delta I_{3}=\frac{e}{\tau} \int_{V_{\mathrm{d}}} \mathrm{e}^{-z / L} p(\mathbf{r}) \mathrm{d} \tau .
\end{aligned}
$$

Let us point out, in agreement with the second critical remark of Pasemann and Donolato in their comment [1] that, formally, the term $\delta p$ entering the flux integral has to be calculated as the limit $\delta p^{-}$at the surface $S_{\mathrm{d}}$ of the $\delta p$ function valid inside the volume $V_{\mathrm{d}}$. However, this quantity $\delta p^{-}$is obviously equal to the limit value $\delta p^{+}$of the $\delta p$ function calculated outside $V_{d}$ since, as schematically shown in figure 1a, any function entering the actual problem is indeed continuous and derivable at any point.

a)

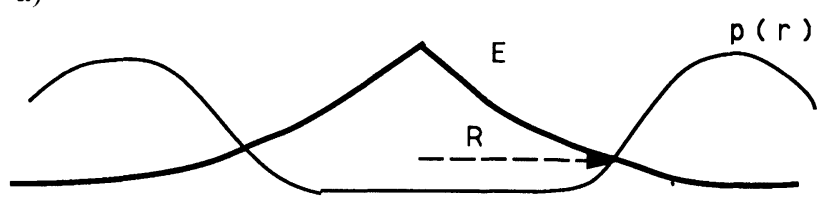

b)

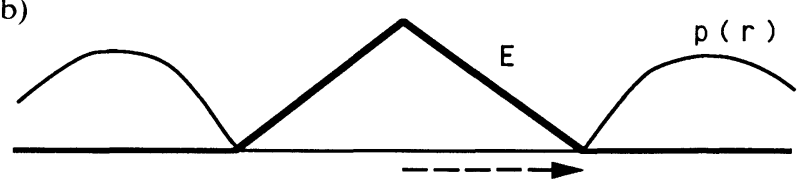

$\mathrm{R}$

Fig. 1. - Variations of the dislocation electric field and of the minority carrier density : a) real situation, b) approximate situation (our model).

In practical cases, the various terms entering expression (9) can no longer be mathematically calculated in a rigourous way. One has, now, to make some physical approximations. In figure 1a it can be noticed that within $V_{\mathrm{d}}$ the $p(\mathbf{r})$ function is essentially negligible because of the presence of the electrical field of large amplitude. Such a situation can be approximated by figure $1 \mathrm{~b}$ where the electrical field does only exist within $V_{\mathrm{d}}$ and where the $p(\mathbf{r})$ function is made equal to zero within $V_{\mathrm{d}}$. In such a model, expression (9) reduces to :

$$
\begin{aligned}
\delta I \cong-\frac{e}{\tau} \int_{V_{\mathrm{d}}} \mathrm{e}^{-z / L} p_{0}(\mathbf{r}) \mathrm{d} \tau- & \\
& -e D \int_{S_{\mathrm{d}}} \mathrm{e}^{-z / L} \operatorname{grad} \delta p^{+}(\mathbf{r}) \mathrm{d} \tau
\end{aligned}
$$

since $\operatorname{div}[\boldsymbol{\sigma E}]=0$ outside $V_{\mathrm{d}}$ and since the quantity $p(\mathbf{r})$, and therefore $\delta \mathbf{j}_{\mathbf{d}}$, are both equal to zero inside $V_{\mathrm{d}}$.

Moreover, the quantity $\delta p^{+}$entering the flux integral of expression (11) has to be calculated by assuming that the $p(\mathbf{r})$ excess carrier distribution vanishes at the cylinder surface surrounding the dislocation. Note also that such a description of the electrical field corresponds to Read's model [6] where the dislocation screening charge is assumed to be a depleted cylinder.

Thus, one of the major objections of Donolato and Pasemann claiming that «bad use is made of equation (18) " of the original paper is a formal mathematical point which we have overcome by 
makıng the physical approximation that the slope of the $p(\mathbf{r})$ function at point $\mathrm{R}$ shown in figure 1a could be evaluated by the slope of the $p(r)$ function shown in figure $1 \mathrm{~b}$. Notice that this approximation is identical to the so-called «depleted region approximation " generally used at the bottom of the space charge region of the Schottky diode when calculating the EBIC current collected by the Schottky diode. Indeed the slope of the $p(r)$ function evaluated by assuming that $p(\mathbf{r})$ vanishes at the collecting plane separating the depleted region from the bulk is not better calculated (see Figs. $2 a$ and b).

a)

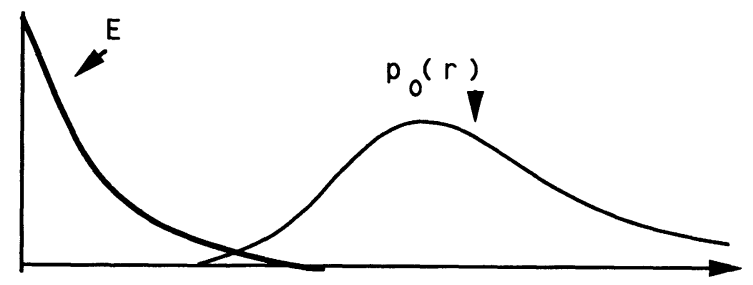

Z

b)

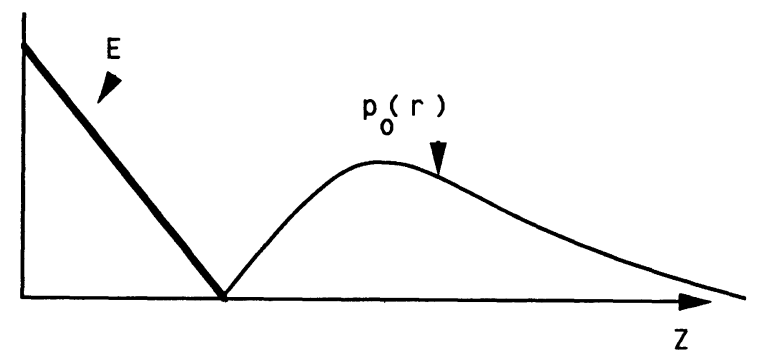

Fig. 2. - Variations of the space charge region (SCR) electric field of a Schottky diode and of the minority carrier density in the bulk and SCR regions. a) real situation, b) approximation of the depleted zone.

The other main objection done by Donolato and Pasemann consists to claim that, owing to the first term of expression (11), our formulation leads to an « absurd " result since a contrast is obtained even in absence of any dislocation. Obviously, this comment is surprising since it is easy to understand that in absence of a dislocation, the volume $V_{\mathrm{d}}$ containing its associated electrical field is equal to zero and therefore the volume integral vanishes too!

The third main comment consists to note that "some of the formula are manifestely incorrect... particularly, formula (36) and the following » since after some integration over the $z$ variable, the resulting formulae do always depend on the quantities $\mathbf{r}-\mathbf{r}^{\prime}$. To this point, we do apologize. Having, before equation (36) of paper [2], made explicitly use of the variable separation, we have not specified that the vectors $\mathbf{r}$ and $\mathbf{r}^{\prime}$ were now polar vectors lying in $(x, y)$ planes. However, little examination of our formula easily shows that we did not have made the confusion Donolato and Pasemann supposed we did.

Donolato and Pasemann also point out that a lot of minor errors have been left in the paper (sign errors and/or omissions of factors...). They are right!

Finally, Donolato and Pasemann claim, contrarily to what we have written, that some previous models also lead to quantitative evaluation of the EBIC contrast (for instance the paper [7] by Pasemann et al.). Indeed, quantitative contrast values can be derived from their model. However, they depend on the choice of $\left(\tau^{\prime}, R\right)$ couples $\left(\tau^{\prime}\right.$ being the reduced lifetime within the cylinder of radius $R$ surrounding the dislocation line). A single choice of such coupled quantities cannot be easily deduced from the physical properties of the dislocation. In contrast, in our model they are no more adjustable parameters since the contrast values only depend on the radius of the screening cylinder which is itself physically determined by the dislocation energy level position, by the characteristics of the host material and the experimental conditions (doping density, temperature a.s.o.).

Thus, contrarily to the conclusions of the Donolato and Pasemann's comment, we believe that our model leads to correct order of magnitude of the EBIC contrast and recommand its use in order to check up, by such local observations, some dislocation properties.
[1] Donolato C. and Pasemann L., Revue Phys. Appl. 25, nं 9 (1990).

[2] Farvacque J. L. and Sieber B., Revue Phys. Appl. 25 (1990) 353.

[3] Wilshaw P. R. and Booker G. R., Microsc. Semicond. Mater. Conf., Oxford, Inst. Phys. Conf. Ser. 7 (1985) 329.
[4] Ferre D., Diallo A. and Farvacque J. L., Revue Phys. Appl. 25 (1990) 177.

[5] Read W. T., Philos. Mag. 45 (1954) 775 et 1119.

[6] Pasemann L., Blumtritt H. and Gleichmann R., Phys. Status Solidi (a) 70 (1982) 197. 